\title{
Preoperative neutrophil-lymphocyte and platelet- lymphocyte ratios as independent predictors of cervical stromal involvement in surgically treated endometrioid adenocarcinoma
}

\author{
This article was published in the following Dove Press journal: \\ OncoTargets and Therapy \\ 15 March 2013 \\ Number of times this article has been viewed
}

\author{
Dan Wang \\ Jia-Xin Yang \\ Dong-Yan Cao \\ Xi-Run Wan \\ Feng-Zhi Feng \\ Hui-Fang Huang \\ Keng Shen \\ Yang Xiang \\ Department of Obstetrics and \\ Gynecology, Peking Union Medical \\ College Hospital, Peking Union \\ Medical College and Chinese Academy \\ of Medical Sciences, Beijing, People's \\ Republic of China
}

Correspondence: Yang Xiang Department of Obstetrics and Gynecology, Peking Union Medical College Hospital, Peking Union Medical College and Chinese Academy of Medical Sciences, Beijing 100730, People's

Republic of China

Tel +86 1065296218

Fax +86 I0 65I2 4875

Email xiangyang65@gmail.com

\begin{abstract}
Background: The purpose of this study was to evaluate the relationship between preoperative inflammatory markers (neutrophil-lymphocyte ratio and platelet-lymphocyte ratio) and cervical stromal involvement in patients with endometrioid adenocarcinoma.

Methods: We studied 318 patients with endometrioid adenocarcinoma who underwent comprehensive surgical staging. We used univariate and multivariate analyses of cervical stromal involvement and receiver-operating curves to calculate optimal cutoff values for neutrophillymphocyte and platelet-lymphocyte ratios to predict cervical stromal involvement.

Results: The presence of cervical stromal involvement was associated with neutrophillymphocyte ratio and platelet-lymphocyte ratio $(P=0.009$ and $P=0.031$, respectively). Multivariate analysis showed that higher neutrophil-lymphocyte and platelet-lymphocyte ratios independently predicted cervical stromal involvement (odds ratio 3.10, 95\% confidence interval $1.10-8.76, P=0.032$, and odds ratio $5.27,95 \%$ confidence interval $1.94-14.35, P=0.001$, respectively). At a threshold of 2.01, the neutrophil-lymphocyte ratio was $71.0 \%$ sensitive and $63.8 \%$ specific for stromal involvement; at a 172.24 threshold, the platelet-lymphocyte ratio was $48.4 \%$ sensitive and $88.9 \%$ specific.

Conclusion: Preoperative neutrophil-lymphocyte and platelet-lymphocyte ratios can help identify the risk of cervical stromal involvement in patients with endometrial cancer. Evaluating these ratios may help select patients who should be particularly watched and tested for cervical stromal involvement.
\end{abstract}

Keywords: neutrophil-lymphocyte ratio, platelet-lymphocyte ratio, endometrioid adenocarcinoma, cervical stromal involvement

\section{Introduction}

Endometrioid adenocarcinoma is the most common gynecological cancer in the western world. ${ }^{1}$ Most endometrial cancer presents with disease limited to the uterus, but $7 \%-15 \%$ of cases present with some cervical stromal involvement. Patients with cervical stromal involvement have significantly worse prognoses than those whose endometrial cancer is confined to the uterine body. Furthermore, cervical stromal involvement is considered to increase the risk of lymph node metastasis. For patients with cervical involvement, radical hysterectomy including parametrectomy and lymphadenectomy may be advocated. The Surveillance Epidemiology and End Results analysis of 932 patients with International Federation of Gynecology and Obstetrics (FIGO) stage II endometrial cancer indicated a better prognosis using 
radical hysterectomy than simple hysterectomy. ${ }^{2}$ For patients undergoing lymphadenectomy, more surgical blood loss, longer surgical time, and high complication rates were reported. ${ }^{3}$ Thus, preoperatively identifying patients at high risk for cervical stromal involvement, and who may need radical surgery and lymphadenectomy is important in terms of both treatment and prognosis. Unfortunately, preoperative evaluation methods, including fractional curettage, transvaginal sonography, and hysteroscopic assessment, are not sensitive enough to detect cervical stromal involvement. ${ }^{4,5}$ Although magnetic resonance imaging is highly sensitive and specific for cervical stromal involvement, it is costly and time-consuming. ${ }^{6}$ Therefore, development of a noninvasive and readily available preoperative test is needed to identify patients with cervical stromal involvement in endometrial cancer.

Inflammation evidently plays an important role in development of cancer and its progression. ${ }^{7}$ Systemic inflammatory markers have been reported to be prognostic and predictive markers in various kinds of cancers. ${ }^{8}$ $\mathrm{C}$-reactive protein is one index of systemic inflammation. However, C-reactive protein is not routinely measured as part of preoperative examination. The neutrophil to lymphocyte ratio (NLR) is another marker of systemic inflammatory response, ${ }^{9}$ and some studies have shown elevated NLR to be a significant prognostic factor in patients with various malignancies. ${ }^{8}$ The platelet to lymphocyte ratio (PLR) is reportedly a significant prognostic indicator in resected pancreatic cancer. ${ }^{10}$ However, little is known about the predictive values of NLR and PLR in endometrial cancer, and only a few studies have evaluated their predictive value for lymph node metastasis in the disease. ${ }^{11}$

To the best of our knowledge, there have been no reports about the predictive value of inflammatory markers for cervical stromal involvement in endometrioid adenocarcinoma. Therefore, in this study, we evaluated the relationship between preoperative inflammatory markers and cervical stromal involvement in endometrioid adenocarcinoma.

\section{Materials and methods}

Retrospective data were collected for patients treated by surgical staging procedures between January 2003 and June 2012 at Peking Union Medical College Hospital. Eligibility criteria for this study included histologically confirmed endometrioid adenocarcinoma, and comprehensive surgical staging, including pelvic or para-aortic lymph node dissection. Patients with second malignancies, hematological disease, inflammatory disease, recombinant granulocyte colony-stimulating factor use, or missing preoperative complete blood cell count or complete blood cell count drawn more than two weeks prior to surgery were excluded. Patients who had undergone prior chemotherapy or pelvic radiation were excluded. Surgical staging consisted of peritoneal cytology, total abdominal hysterectomy, bilateral salpingooophorectomy, and systemic pelvic lymphadenectomy with or without para-aortic lymphadenectomy. Radical hysterectomy was performed when cervical stromal involvement was suspected. Pelvic lymphadenectomy includes the common iliac and obturator nodes above the obturator nerve.

Patients were staged according to the 2009 FIGO guidelines. Endometrial cancer was graded according to FIGO classification. Complete blood cell count, age, stage, histologic grade, lower uterine segment involvement, presence of lymphovascular invasion, depth of myometrial invasion, cervical stromal involvement, positive peritoneal cytology, and pelvic or para-aortic lymph node status were reviewed from patients' medical records. The study was approved by the institutional review board of Peking Union Medical College Hospital.

The NLR was defined as the absolute neutrophil count divided by the absolute lymphocyte count; similarly, PLR was defined as the absolute platelet count divided by the absolute lymphocyte count.

\section{Statistical analyses}

Categorical variables were analyzed using Pearson's Chisquare test and Fisher's Exact test. Continuous variables were expressed as the mean \pm standard deviation and compared using the Student's $t$-test. Univariate and multivariate analyses were used to assess relationships between the various factors and cervical stromal involvement. Odds ratios (OR) with $95 \%$ confidence interval $(\mathrm{CI})$ were calculated by univariate or multivariate analysis using the logistic regression test. Sensitivity and specificity for different NLR and PLR cutoffs were calculated with receiver-operating curves. Receiver-operating curve analysis was plotted to investigate optimal cutoff values that maximized sensitivity and specificity. All statistical analyses were performed using the Statistical Package for Social Sciences version 16.0 software (SPSS Inc, Chicago, IL, USA). $P<0.05$ was considered to be statistically significant.

\section{Results}

Three hundred and eighteen patients (mean age 55.14 years) with endometrioid adenocarcinoma who met the criteria were 
included in the study. The distribution of surgical FIGO stages was 254 in stage I, 19 in stage II, 41 in stage III, and four in stage IV. Of the 318 patients, 31 (9.75\%) presented with cervical stromal involvement.

Table 1 shows the clinicopathological features of the patients with and without cervical stromal involvement. Cervical stromal involvement was more common in the presence of advanced FIGO stage, higher grades of tumor, lower uterine segment involvement, deep myometrial invasion, positive lymph node status, and positive adnexal involvement $(P<0.001, P=0.035, P<0.001, P=0.002$, $P<0.001$, and $P=0.024$, respectively). Logistic regression analysis found that only lower uterine segment involvement and positive lymph node status were independent risk factors for cervical stromal involvement (OR 9.18, 95\% CI 3.17-26.58, $P<0.001$, and OR 4.82, 95\% CI 1.21-19.21, $P=0.026$, respectively).

Mean counts of white blood cell subtypes, platelets, NLR, and PLR are shown in Table 2. Mean values for white blood cells, neutrophils, platelets, NLR, and PLR were increased in the positive group $(P=0.012, P<0.001, P=0.001$, $P=0.009$, and $P=0.031$, respectively). There was no

Table I Patient characteristics and pathological findings in patients with or without cervical stromal involvement

\begin{tabular}{|c|c|c|c|}
\hline Characteristics & $\begin{array}{l}\text { CSI-negative } \\
(n=287)\end{array}$ & $\begin{array}{l}\text { CSI-positive } \\
(n=31)\end{array}$ & $P$ \\
\hline Mean age (SD) & $58.84 \pm 10.16$ & $52.90 \pm 9.99$ & 0.312 \\
\hline FIGO stage, n (\%) & & & $<0.001$ \\
\hline I & $254(88.5 \%)$ & $0(0.0 \%)$ & \\
\hline II-IV & 33 (11.5\%) & $31(100.0 \%)$ & \\
\hline Grade & & & 0.035 \\
\hline I & 159 (55.4\%) & $14(45.2 \%)$ & \\
\hline II & 99 (34.5\%) & $9(29.0 \%)$ & \\
\hline III & $29(10.1 \%)$ & $8(25.8 \%)$ & \\
\hline LUSI (n= 3|7) & & & $<0.001$ \\
\hline Negative & I 93 (67.5\%) & $6(19.4 \%)$ & \\
\hline Positive & 93 (32.5\%) & 25 (80.6\%) & \\
\hline Myometrial invasion & & & 0.002 \\
\hline$<\mathrm{I} / 2$ & $220(76.7 \%)$ & $16(51.6 \%)$ & \\
\hline$\geq \mathrm{I} / 2$ & $67(23.3 \%)$ & $15(48.4 \%)$ & \\
\hline LVSI & & & 0.397 \\
\hline Negative & $263(91.6 \%)$ & $27(81.0 \%)$ & \\
\hline Positive & $24(8.4 \%)$ & $4(12.9 \%)$ & \\
\hline Lymph node & & & $<0.001$ \\
\hline Negative & $273(95.1 \%)$ & $24(77.4 \%)$ & \\
\hline Positive & 14 (4.9\%) & 7 (22.6\%) & \\
\hline Adnexal involvement & & & 0.024 \\
\hline Negative & 276 (96.2\%) & 27 (87.1\%) & \\
\hline Positive & II (3.8\%) & 4 (I2.9\%) & \\
\hline
\end{tabular}

Abbreviations: CSI, cervical stromal involvement; FIGO, International Federation of Gynecology and Obstetrics; LUSI, lower uterine segment involvement; LVSI, lymphovascular space invasion; SD, standard deviation.
Table 2 Mean white blood cell subtype counts, neutrophil-tolymphocyte ratio, and platelet-to-lymphocyte ratio in study subjects

\begin{tabular}{lccl}
\hline & $\begin{array}{l}\text { CSI-negative } \\
(\mathbf{n}=\mathbf{2 8 7})\end{array}$ & $\begin{array}{l}\text { CSI-positive } \\
(\mathbf{n}=\mathbf{3} \mathrm{I})\end{array}$ & $\boldsymbol{P}$ \\
\hline WBC & $6.26 \pm 1.62$ & $7.04 \pm 1.67$ & 0.012 \\
Neutrophil & $3.72 \pm 1.21$ & $4.65 \pm 1.31$ & $<0.00 \mathrm{I}$ \\
Lymphocyte & $2.11 \pm 0.64$ & $1.91 \pm 0.62$ & 0.104 \\
Platelet & $241.76 \pm 61.63$ & $314.19 \pm 105.72$ & 0.001 \\
NLR & $1.86 \pm 0.67$ & $2.71 \pm 1.67$ & 0.009 \\
PLR & $122.17 \pm 41.54$ & $193.48 \pm 175.09$ & 0.031 \\
\hline
\end{tabular}

Note: Values are expressed as the mean \pm standard deviation.

Abbreviations: CSI, cervical-stromal involvement; NLR, neutrophil-lymphocyte ratio; PLR, platelet-lymphocyte ratio; WBC, white blood count.

significant difference in the mean value for lymphocytes between the two groups.

The cutoff values for preoperative NLR and PLR predicting the presence of cervical stromal involvement along with receiver-operating curves are shown in Figure 1. An optimal cutoff value that maximized the sum of sensitivity and specificity in the receiver-operating curve was used. The best cutoff value for NLR was 2.01, which was $71.0 \%$ sensitive and $63.8 \%$ specific for the presence of cervical stromal involvement. The best cutoff value for PLR was 172.24 , which was $48.4 \%$ sensitive and $88.9 \%$ specific. NLR and PLR were independent predictors of cervical stromal involvement in patients with endometrial cancer when using 2.01 (NLR) and 172.24 (PLR) as threshold values (OR 3.10, 95\% CI 1.10-8.76, $P=0.032$, and OR 5.27, 95\% CI 1.94-14.35, $P=0.001$, respectively, Table 3).

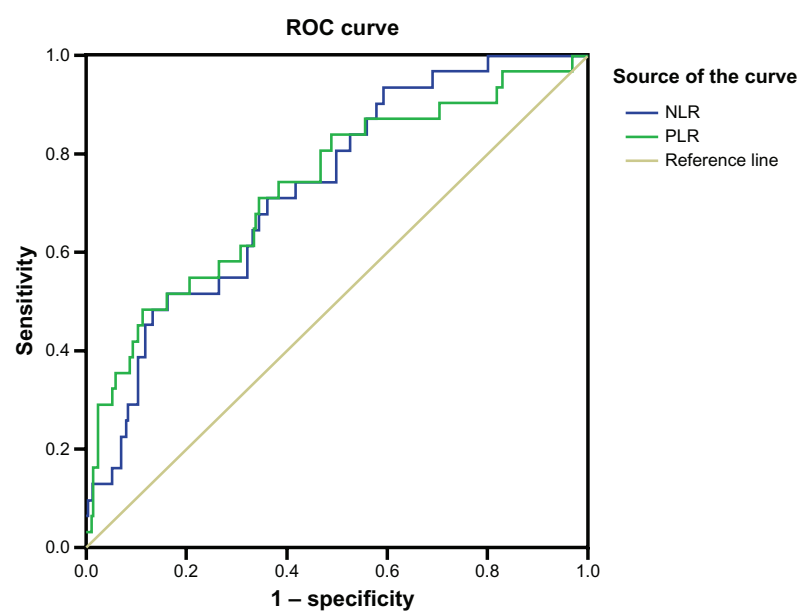

Figure I Receiver-operating curve for the relationship between NLR and PLR and cervical involvement.

Notes: Areas under the curve for NLR and PLR are 0.73 I $(95 \%$ confidence interval $0.646-0.817, P<0.001)$ and 0.732 (95\% confidence interval $0.632-0.832$, $P<0.00$ I), respectively.

Abbreviations: NLR, neutrophil-lymphocyte ratio; PLR, platelet-lymphocyte ratio. 
Table 3 Effect of univariate and multivariate variables on the presence of cervical stromal involvement in patients with endometrial cancer

\begin{tabular}{|c|c|c|c|c|c|c|}
\hline \multirow[t]{2}{*}{ Variables } & \multicolumn{3}{|c|}{ Univariate } & \multicolumn{3}{|c|}{ Multivariate } \\
\hline & OR & $95 \% \mathrm{Cl}$ & $P$ & OR & $95 \% \mathrm{Cl}$ & $P$ \\
\hline \multicolumn{7}{|l|}{$\geq \mathrm{CO}$ versus $<\mathrm{CO}$} \\
\hline NLR* & 4.24 & I.88-79.54 & $<0.001$ & 3.10 & $1.10-8.76$ & 0.032 \\
\hline PLR* & 7.47 & $3.38-16.54$ & $<0.001$ & 5.27 & $1.94-14.35$ & 0.001 \\
\hline \multicolumn{7}{|l|}{ Grade } \\
\hline I & Refer & & & & & \\
\hline ॥ & 1.03 & $0.43-2.48$ & 0.943 & 0.37 & $0.12-1.17$ & 0.090 \\
\hline III & 3.13 & $1.21-8.14$ & 0.019 & 1.45 & $0.39-5.40$ & 0.583 \\
\hline LUSI, positive versus negative & 8.65 & $3.43-21.80$ & $<0.001$ & 9.18 & $3.17-26.58$ & $<0.001$ \\
\hline $\begin{array}{l}\text { Myometrial invasion } \geq \mathrm{I} / 2 \\
\text { versus }<\mathrm{I} / 2\end{array}$ & 3.08 & $1.45-6.55$ & 0.004 & 1.20 & $0.44-3.26$ & 0.715 \\
\hline LVSI, positive versus negative & 1.62 & $0.52-5.03$ & 0.401 & - & & \\
\hline Lymph node positive versus negative & 5.69 & $2.10-15.44$ & 0.001 & 4.82 & $|.21-19.2|$ & 0.026 \\
\hline $\begin{array}{l}\text { Adnexal involvement, positive } \\
\text { versus negative }\end{array}$ & 3.72 & $1.11-12.47$ & 0.034 & 1.41 & $0.27-7.46$ & 0.685 \\
\hline
\end{tabular}

Notes: The CO for NLR is 2.0I; the CO for PLR is 172.24. *After multivariate analysis, the differences were still significant.

Abbreviations: CO, cutoff value; NLR, neutrophil-lymphocyte ratio; PLR, platelet-lymphocyte ratio; LUSI, lower uterine segment involvement; LVSI, lymphovascular space invasion.

\section{Discussion}

To our knowledge, this is the first study to describe an association between preoperative NLR and PLR and cervical stromal involvement in endometrioid carcinoma. Our results show that preoperative NLR and PLR are independent predictors of cervical stromal involvement in the disease.

Endometrial cancer with cervical stromal involvement has a worse prognosis than cancer confined to the corpus uteri. ${ }^{12}$ Cervical stromal involvement should also be considered a risk factor for lymph node metastasis; in our cases, lymph node metastasis rates were $22.6 \%$ and $4.9 \%$, respectively, in cases with and without cervical stromal involvement. For patients with cervical stromal involvement, radical hysterectomy and systemic lymphadenectomy were required to identify extension of disease and the type of adjuvant therapy. These patients may also benefit from primary radical hysterectomy, with removal of the uterus, cervix, parametrium, and upper vagina. ${ }^{13} \mathrm{~A}$ publication by the US National Institute of Cancer reported that patients with stage II disease had better survival after radical surgery than after simple hysterectomy. ${ }^{2}$ Therefore, preoperative evaluation of cervical stromal involvement may be needed to determine the best surgical approach.

Different preoperative diagnostic tools were used to evaluate the cervical stromal involvement in endometrial cancer. Transvaginal sonographies followed by endometrial sampling or diagnostic hysteroscopy with biopsy are often used. However, there has been no consensus as to the best predictor of cervical stromal involvement. ${ }^{14}$ Endocervical curettage as a part of a procedure that includes dilation and curettage under general anesthesia is costly and may lead to complications in elderly patients. ${ }^{6}$ Magnetic resonance imaging with contrast enhancement is highly sensitive and specific in detecting cervical stromal involvement, but is also costly, time-consuming, and often unavailable, and requires contrast agents that can cause allergic reactions. ${ }^{15}$

The association between cancer and inflammation was discovered over a century ago. Cancer-related inflammation causes suppression of antitumor immunity by recruiting regulatory $\mathrm{T}$ cells and activating chemokines, which results in tumor growth and metastasis. ${ }^{10}$ The presence of both neutrophilia and thrombocytosis tends to represent a nonspecific response to cancer-related inflammation. ${ }^{10}$ The mechanism between preoperative leukocytosis and neutrophilia and cancer remains unclear. However, cancer has been shown to produce myeloid growth factors, such as granulocyte colony-stimulating factor, interleukin (IL)-1, IL-6, and tumor necrosis factor-alpha, which may influence tumor-related leukocytosis and neutrophilia. ${ }^{8}$ Neutrophilia promotes development and progression of cancer by providing a suitable environment and secreting most circulating vascular endothelial growth factors. ${ }^{16}$ In a study by Worley et al in patients with leukocytosis in endometrial carcinoma, presentations accompanied by pathologic factors associated with poorer prognoses and with leukocytosis were independently associated with increased risk of death. ${ }^{17}$ Tavares-Murta et al showed that in patients with cervical cancer, those with advanced cancers had 
significantly higher frequency of leukocytes alterations than did those with preinvasive cancers, and that neutrophilia was the best indicator of cancer invasiveness. ${ }^{18}$

The underlying mechanism for the relationship between platelet expression and the biologic behavior of cancer cells is unclear. Thrombocytosis may be mediated by megakaryocytes and their precursors as a result of stimulation by proinflammatory mediators such as IL-1, IL-2, and IL-6. ${ }^{19}$ An elevated platelet count may simply be an additional indicator of the severity of inflammation. ${ }^{10}$

A major risk factor for endometrial cancer is a lowgrade inflammatory state. Chronic inflammation, through its association with insulin resistance and estrogen production, might mediate the obesity-related increase in risk of endometrial cancer. ${ }^{20}$ Dossus et al report that elevated prediagnostic levels of the inflammatory markers C-reactive protein, IL6, and IL1Ra were associated with an increased risk of endometrial cancer. ${ }^{20}$ In a multicenter study, elevated serum C-reactive protein was associated with a less favorable prognosis in patients with surgically treated endometrial cancer. ${ }^{21}$ However, C-reactive protein is not routinely measured in clinic. Elevated NLR and PLR are likely to represent nonspecific responses to cancer-related inflammation. Many studies have shown elevated NLR and PLR to be prognostic factors in various kinds of cancers. ${ }^{8,10}$ Although NLR and PLR have been associated with lymph node metastasis in endometrial cancer, ${ }^{11}$ we have seen no previous report on the relationship between inflammatory markers and cervical stromal involvement.

Our most interesting finding here is that elevated preoperative NLR and PLR were correlated with an increased risk of cervical stromal involvement, which was noted after multivariate analysis. Cervical stromal involvement was significantly associated with both elevated NLR and PLR (OR 3.10, 95\% CI 1.10-8.76, $P=0.032$, and OR 5.27, 95\% CI 1.94-14.35, $P=0.001$, respectively).

Although preoperative assessment of cervical stromal involvement using magnetic resonance imaging is highly sensitive and specific, this method is also expensive; however, preoperative leukocyte, neutrophil, and platelet counts are routinely performed for such patients. Thus, NLR and PLR may be an accessible preliminary tool to select patients for further evaluation of cervical stromal involvement, eg, via magnetic resonance imaging.

The current investigation has the limitations common to all retrospective studies. Incomplete blood counts might have falsely elevated inflammatory marker values. In addition, C-reactive protein is not routinely measured at our center, and its association with cervical stromal involvement in endometrial cancer could not be analyzed in our study.

In conclusion, this report shows, for the first time, a relationship between systemic inflammatory markers and cervical stromal involvement in endometrial cancer. Preoperative NLR and PLR independently predict cervical stromal involvement in the disease, and may help select patients who should be especially evaluated for cervical stromal involvement.

\section{Disclosure}

The authors report no conflicts of interest in this work.

\section{References}

1. Doll A, Abal M, Rigau M, et al. Novel molecular profiles of endometrial cancer - new light through old windows. J Steroid Biochem Mol Biol. 2008;108(3-5):221-229.

2. Cornelison TL, Trimble EL, Kosary CL. SEER data, corpus uteri cancer: treatment trends versus survival for FIGO stage II, 1988-1994. Gynecol Oncol. 1999;74(3):350-355.

3. Larson DM, Johnson K, Olson KA. Pelvic and paraaortic lymphadenectomy for surgical staging of endometrial cancer: morbidity and mortality. Obstet Gynecol. 1992;79(6):998-1001.

4. Fotiou S, Vlahos N, Kondi-Pafiti A, et al. Intraoperative gross assessment of myometrial invasion and cervical stromal involvement in endometrial cancer: role of tumor grade and size. Gynecol Oncol. 2009;112(3):517-520.

5. Lo KW, Cheung TH, Yim SF, Chung TK. Preoperative hysteroscopic assessment of cervical invasion by endometrial carcinoma: a retrospective study Gynecol Oncol. 2001;82(2):279-282.

6. Haldorsen IS, Berg A, Werner HM, et al. Magnetic resonance imaging performs better than endocervical curettage for preoperative prediction of cervical stromal invasion in endometrial carcinomas. Gynecol Oncol. 2012;126(3):413-418.

7. Mantovani A, Allavena P, Sica A, et al. Cancer-related inflammation. Nature. 2008;454(7203):436-444.

8. Kwon HC, Kim SH, Oh SY, et al. Clinical significance of preoperative neutrophil-lymphocyte versus platelet-lymphocyte ratio in patients with operable colorectal cancer. Biomarkers. 2012;17(3):216-222.

9. Zahorec R. Ratio of neutrophil to lymphocyte counts - rapid and simple parameter of systemic inflammation and stress in critically ill. Bratisl Lek Listy. 2001;102(1):5-14.

10. Bhatti I, Peacock O, Lloyd G, Larvin M, Hall RI. Preoperative hematologic markers as independent predictors of prognosis in resected pancreatic ductal adenocarcinoma: neutrophil-lymphocyte versus platelet-lymphocyte ratio. Am J Surg. 2010;200(2):197-203.

11. Suh DH, Kim HS, Chung HH, et al. Pre-operative systemic inflammatory response markers in predicting lymph node metastasis in endometrioid endometrial adenocarcinoma. Eur J Obstet Gynecol Reprod Biol. 2012;162(2):206-210.

12. Morrow CP, Bundy BN, Kurman RJ, et al. Relationship between surgical-pathological risk factors and outcome in clinical stage I and II carcinoma of the endometrium: a Gynecologic Oncology Group study. Gynecol Oncol. 1991;40(1):55-65.

13. Cohn DE, Woeste EM, Cacchio S, et al. Clinical and pathologic correlates in surgical stage II endometrial carcinoma. Obstet Gynecol. 2007;109(5):1062-1067.

14. Almog B, Gutman G, Lessing JB, Grisaru D. Prediction of cervical stromal involvement in endometrial cancer by hysteroscopy. Arch Gynecol Obstet. 2007;275(1):45-48. 
15. Akbayir O, Corbacioglu A, Numanoglu C, et al. Preoperative assessment of myometrial and cervical invasion in endometrial carcinoma by transvaginal ultrasound. Gynecol Oncol. 2011;122(3):600-603.

16. Kusumanto YH, Dam WA, Hospers GA, Meijer C, Mulder NH. Platelets and granulocytes, in particular the neutrophils, form important compartments for circulating vascular endothelial growth factor. Angiogenesis. 2003;6(4):283-287.

17. Worley MJ Jr, Nitschmann CC, Shoni M, et al. The significance of preoperative leukocytosis in endometrial carcinoma. Gynecol Oncol. 2012;125(3):561-565.

18. Tavares-Murta BM, Mendonca MAO, Duarte NL, et al. Systemic leukocyte alterations are associated with invasive uterine cervical cancer. Int J Gynecol Cancer. 2010;20(7):1154-1159.
19. Klinger MH, Jelkmann W. Role of blood platelets in infection and inflammation. J Interferon Cytokine Res. 2002;22(9):913-922.

20. Dossus L, Rinaldi S, Becker S, et al. Obesity, inflammatory markers, and endometrial cancer risk: a prospective case-control study. Endocr Relat Cancer. 2010;17(4):1007-1019.

21. Schmid M, Schneitter A, Hinterberger S, et al. Association of elevated $\mathrm{C}$-reactive protein levels with an impaired prognosis in patients with surgically treated endometrial cancer. Obstet Gynecol. 2007;110(6): $1231-1236$.

\section{Publish your work in this journal}

OncoTargets and Therapy is an international, peer-reviewed, open access journal focusing on the pathological basis of all cancers, potential targets for therapy and treatment protocols employed to improve the management of cancer patients. The journal also focuses on the impact of management programs and new therapeutic agents and protocols on

\section{Dovepress}

patient perspectives such as quality of life, adherence and satisfaction. The manuscript management system is completely online and includes a very quick and fair peer-review system, which is all easy to use. Visit http://www.dovepress.com/testimonials.php to read real quotes from published authors. 